\title{
BARYON SPECTROSCOPY AND THE OMEGA MINUS*
}

N. P. Samios

Brookhaven National Laboratory

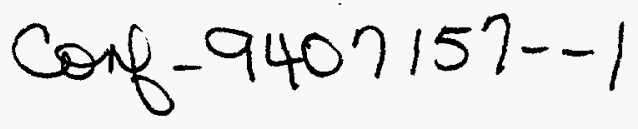

Upton, New York 11973

In my talk today, I will mainly discuss baryon resonances with emphasis on the discovery of the $\Omega$. However, for completeness, I will also present some data on the meson resonances which together with the baryons led to the uncovering of the SU(3) symmetry of particles and ultimately to the concept of quarks. This period of particle physics was characterized by a strong interplay between theory and experiment. Experiments were numerous and the phenomenology kept pace with the forthcoming results. The early experimental work was performed at cyclotrons, Berkeley, Chicago, Columbia, Carnegie Tech, Rochester, etc. then moving on to the so-called doughnut machine at BNL, LBL, CERN and Cornell. The analysis of the experimental results was greatly aided by the introduction of Lee-Yang test function, Dalitz plots, the Jackson angle, the Treiman-Yang angle, etc. A consequence of this close interaction was the rapid progress in categorizing the large number of meson and baryon resonances. Furthermore, most of the early characterizations were correct, the existence and properties of resonances were validated by later experiments. The search for hadronic constituents began with cosmic rays and by the early 50 's several of the baryons and a few of the mesons had been found. Beyond the proton (p) and neutron (n), the $\Lambda$ and $\Sigma^{+}$and $\Sigma^{-}$were first observed in the cloud chamber studies conducted on mountain tops and the $\Sigma^{-}, \Sigma^{\circ}$ and $\Sigma^{\circ}$ were found in the early bubble chamber experiments carried out at accelerators. In retrospect the composition of the ground state baryon octet was in hand early in these investigations. The meson area was more complicated, the pion in all its forms $\left(\pi^{+}, \pi^{-}, \pi^{9}\right)$ was well known. However, it took many years of painstaking work to establish that the myriad of reported particles with the names $\tau$, $\theta, \mathrm{K} \mu_{3}, \mathrm{Ke}, \chi$ etc. were all just the $\mathrm{K}$ meson comprised of two doublets 
$\left(\mathrm{K}^{+} \mathrm{K}^{\circ}\right)$. It was referring to this situation that Fermi made his remark, "Young man, if I could remember the names of all these particles I would have become a botanist!" Imagine what he would have said in the ensuing years when the hundreds of new particles were reported. It is interesting to note that it was Fermi's group that started this explosion with the $\Delta$ resonance, the first short lived state, lifetime $-10^{-23} \mathrm{sec}$. From a phase shift analysis of pion nucleon interaction at low energies, they speculated that a resonant state, the $\Delta$, should exist at a higher mass, ${ }^{1}$ which was indeed found at BNL's newly inaugurated Cosmotron. The two major methods for exploring this rich domain of particles are formation experiments and production experiments. The former have been very effective in uncovering baryon resonances $N, \Delta, \Lambda, \Sigma$ 's while the latter have been extremely productive in the meson resonance arena. Such reactions are illustrated in Fig. 1 and some successes in the ensuing figures. The study of $\pi^{+} p$ and $\pi p$ total cross sections as a function of energy (or mass of the system) clearly established the existence of many $N$ and $\Delta$ resonances (Fig. 2). Similar peaks are observed in $\mathrm{K} p$ cross sections announcing the presence of numerous $\Lambda$ and $\Sigma$ states (not shown). The early evidence for the $\rho,{ }^{2} K(890),{ }^{3} \Sigma(1385),{ }^{4}$ $\omega,{ }^{5}$ and $\eta^{6}$ are shown in Figs. 3 and 4. They all stand out to the naked eye as clear bumps above the background, clearly attesting to the fact that these ground states were produced with appreciable cross sections. In fact, it is interesting to note that the experiment that found the $\eta^{6}$ could easily have also discussed the $\omega$.

The resonance situation as of August 1961 is noted in Table I; limited number of states, masses and widths reasonably well established but with spin parities poorly known. Besides determining the spectroscopy of resonances, a great deal of effort was expended in the question of the relative parity of states, in particular the $\Sigma-\Lambda$ parity and the $K \Lambda N$ parity. In retrospect I am amazed at the amount of energy and resources, debates and controversy that surrounded these issues, mainly the $\Sigma-\Lambda$ relative parity, that are now taken for granted. I will 


\section{DISCLAIMER}

Portions of this document may be illegible in electronic image products. Images are produced from the best available original document. 
just mention the U.S. east and west coast efforts in looking for cusp effects in pion nucleon associated production of $\Lambda \theta$ as suggested by Dalitz and Baz-Okun. This approach didn't work beause of the presence of $d$ waves in addition to $s$ and $p$ waves. The complicated and probably correct deduction of even $\Sigma-\Lambda$ parity by Tripp and collaborators in the detailed study of the $\Lambda(1520)$ decay. There was even a theoretical letter on eight experimental reasons why the $\Sigma-\Lambda$ parity was odd, all wrong. The solution of even parity was demonstrated in the Dalitz decay of the $\Sigma^{0}$ into $\Lambda^{0} \mathrm{e}^{+} \mathrm{e}^{-}$. The equally important issue of the $\mathrm{K} \Lambda \mathrm{N}$ relative parity was resolved to most people's satisfaction from the study of hypernuclear production in $\mathrm{K}^{-}$He interactions.

The year 1962 was especially significant, especially the CERN International Conference on High Energy Physics. It was at this meeting that we presented evidence for both the $\varphi(1020)^{7}$ and the $\Xi(1530)$ see Figs. 5 and 6 . The importance of the $\varphi$ is that it completed the $\mathrm{J}^{\mathrm{P}}=1^{-}$nonet (the other members being the $p, \omega$ and $K(895)$ and whose $\varphi$ mass was such as to achieve ideal mixing

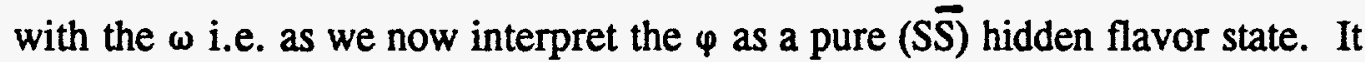
wasn't until 1964 that we presented evidence for the ninth pseudo scaler meson the $\eta^{\prime}(960){ }^{8}$ The unveiling of the $\Xi(1530)$ had even greater consequences. The $\Delta(1238)$ and $\Sigma(1385)$ were well known, the former definitely being $3 / 2^{+}$ while the later was likely $3 / 2^{+}$. This new cascade state with unknown spin parity fit nicely into Gell-Mann's SU(3) scheme' where the members of the decimet (10) would obey an equal mass spacing rule and more important would predict a singlet member at a mass of $1680 \mathrm{MeV}$ ! The $\Omega$. It was a bold conjecture which Murray unveiled (at least to me) at this conference. The SU(3) symmetry was badly broken with the evidence for very few multiplets, however this decimet $\mathrm{J}^{\mathrm{P}}=3 / 2^{+}$was beautiful in its precise predictions both for the mass and decay modes of this conjectured $\Omega^{-}$particle. Since the combined mass of $\Xi(1320) \mathrm{K}(495)$ is more than the expected $\Omega^{\circ}$ mass of 1680 deduced from the equal spacing mass rule it cannot decay strongly, therefore it must 
decay weakly with a corresponding lifetime of $10^{-10} \mathrm{sec}$ i.e. it should traverse a few centimeters before decaying--a visible track in a bubble chamber. The possible decay modes are three; namely:

$\Omega^{-} \rightarrow \Xi^{\cdot} \pi^{\circ}$ double break

$\Omega^{-} \rightarrow \Xi^{\circ} \pi^{-}$non-pointing $\Lambda^{\circ}$

$\Omega^{-} \rightarrow \Lambda \mathrm{K}^{-} \quad \mathrm{K}^{-}$instead of $\pi^{\prime}$ from $\Xi^{-} \rightarrow \Lambda \pi^{-}$decay.

As we now know, the relative branching ratios are 8, 24 and $68 \%$ respectively. To produce the $\Omega^{-}$(strangeness -3 ) it was clearly advantageous to use an incoming $\mathrm{K}^{-}$beam (having one initial strangeness -1 ). In fact we, at BNL, were in the process of designing such a beam for use with the 80 -in. hydrogen bubble chamber which was under construction by the Shutt group. Our thinking was rather straightforward and built on our previous successes. We had used a 2 $\mathrm{GeV} / \mathrm{c} \mathrm{K}^{-}$beam with the 20 -in. hydrogen bubble chamber and were able to produce an abundance of strange particles and in particular $\Xi$ particles with strangeness -2. A straightforward extrapolation suggested that a higher energy $\mathrm{K}^{-}$beam would be even better, and we had settled on $5 \mathrm{GeV} / \mathrm{c}$, since this was the highest energy separated beam technically feasible. As it fortuitously turned out this was well above the threshold energy needed to produce $\Omega^{\circ}$ and the two associated $\mathrm{K}$ mesons, namely $3.2 \mathrm{GeV} / \mathrm{c}$. The program was therefore set, what was required was competent execution. I should remark that there wasn't unanimity to this approach. There were other symmetry schemes in vogue, $\mathrm{G}(2)$ et al. and the baryon ground state was not in good shape, remember the $\Sigma \Lambda$ parity issue. If the $\Sigma-\Lambda$ parity were odd, then the baryon octet is not viable and in particular SU(3) is in trouble. In addition, the AGS was a relatively young machine, it became operational in 1960-1961 and the competition for running time and resources was ferocious. Remember that the second neutrino had just been deciphered in 1962, a whole neutrino program was in full swing as well as programs in the study of weak decays and the strong interactions-dynamics as well as spectroscopy. 
This was then the setting, a relatively new machine, the AGS, a completely new bubble chamber, the 80 -in., and a new complex separated beam line--all pushing the limits of the then present technology. The problems that arose were numerous, but they all were solved, one by one. The bubble chamber developed optical problems, the beam line had deficiences and solutions had to be devised and were devised to overcome them. This, of course, is not new in experimental physics, and it is the case with talented and dedicated people that one succeeds, and this was such a case. The group persevered and we accumulated $\geq 100,000$ bubble chamber pictures with 5-10 $\mathrm{K}^{-}$per picture and proceeded to scan them. Of course, this was another area where scanning tables, measuring machines and computer programs had to be developed for this new chamber--and they were. In order to expedite the process, we had a physicist scanning and on shift at all times. Therefore, whenever a $\Omega^{\circ}$ candidate appeared or for that matter any unusual event pattern appeared, what should be done could be resolved in an expert and expeditious manner. It therefore transpired that it was on my shift, on my scanning table that this historical and beautiful event appeared. The famous event is shown in Fig. 7. The two features that caught my attention was that the decay $\Lambda^{\circ}$ did not appear to come from the decaying vertex (3) and the momentum of the decaying track (4) was larger than allowed by $\Xi^{-}$decay. Both these signatures were indicative of an $\Omega^{-}$ decaying into $\Xi^{\circ} \pi^{-}$with the subsequent decay of the $\Xi^{\circ}$ into $\Lambda^{\circ}$ and a $\pi^{\circ}$. I immediately flagged it as a very interesting event to be measured in detail the next morning. While this event was being measured the next day, several of us were present and discussing the candidate when someone noticed an electronpositron pair (8) and then jokingly someone also commented on the possibility of a second one and within a minute indeed the second one was found (7). This was incredible because of the radiation length in liquid hydrogen is of the order of ten meters, therefore the probability of two $\gamma$ rays materializing is of the order of $10^{-3}$ ! After the event was measured accurately, 1 immediately did a 
rough measurement with templates and protractors on the scanning table and was able to reconstruct the $\pi^{\circ}$ from the two $\gamma^{\prime} s$, the $\Xi^{\circ}$ from the $\Lambda^{\circ}$ and $\pi^{\circ}$ and the $\Omega^{\circ}$ from the $\Xi^{\circ}$ and $\pi^{\circ}$. The rest was details and history. ${ }^{10}$ This first event was therefore an example of $\Omega^{\circ}$ prediction and decay, the sequence being:

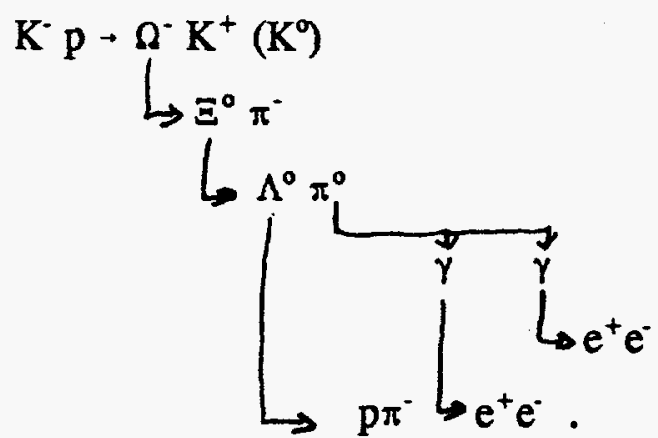

The $\Omega$ mass was determined to be $1686 \pm 12 \mathrm{MeV}$ and the lifetime $0.7 \times 10^{-10}$ sec. In four months when the systematics of the chamber, magnetic field, optics, etc. were better understood the mass value became $1675.6 \pm 5.4 \mathrm{MeV}$, the central value and error both reduced. For historical completeness, I include the second confirming event which we found in September 1964, again containing an unexpected feature. This event is shown in Fig. 8. In this case all the particles are visible in the bubble chamber. The unusual feature in this instance is the visible decay of the $\mathrm{K}^{-}$into three charged pions, a probability of $5 \%$. The interpretation of the event is

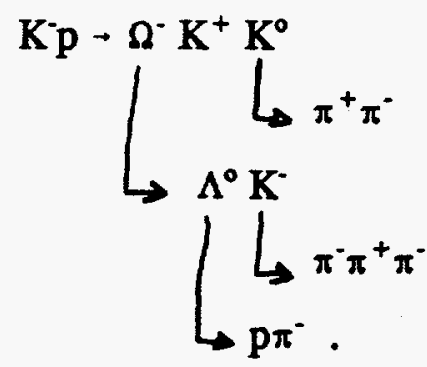

The $\Omega^{-}$mass was determined to be $1674 \pm 3 \mathrm{MeV}$ and the lifetime $1.4 \times 10^{-10}$ sec. By February 1968 we had accumulated eight $\Omega^{-}$events and measured the mass to be $1673.3 \pm 1.0 \mathrm{MeV}$. By the year 1990 the world's value was $\mathrm{m}=$ $1672.43 \pm .32 \mathrm{MeV}$, not bad. For completeness we note that in 1991 experiment E756 at Fermilab had accumulated 143,000 events and the magnetic 
moment of the $\Omega^{-}$was measured to be $-1.94 \mu_{\mathrm{N}}$ for $\mathrm{J}^{\mathrm{P}}=3 / 2^{+}$.

The discovery of the $\Omega^{-}$gave great credence to the SU(3) symmetry scheme of particles. In the next few years many of the mesonic and baryonic multiplets were established. Several nonets were deciphered among the mesons, namely

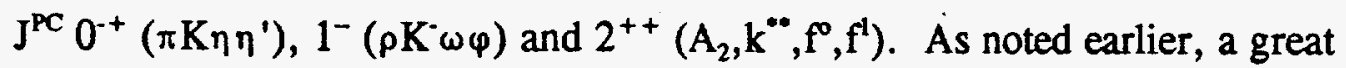
deal of effort by many groups was expended to achieve this level of codification. The existence of the nonet structure for mesons was especially important, as will be discussed shortly, and it is interesting to note that we had the pleasure of finding the ninth member of each of the above monets, namely the $\eta^{1}, \varphi$ and $f^{\prime}$ singlets.

In the baryon domain many octets and several decimets emerged. The octets were $\mathrm{J}^{\mathrm{P}} 1 / 2^{+}, 3 / 2^{-}, 5 / 2^{+}$and $7 / 2^{-}$with the deciments, the $3 / 2^{+}$and $7 / 2^{+}$. Among this large number of resonant states there were no candidates for the $\overline{10}$ or 27 representations, just singlets, octets, nonets and decimets. Such states had been searched for, such as $\mathrm{K}^{+} \mathrm{K}^{+}$meson or $\Xi^{-} \pi^{+} \pi^{+}$baryon but with negative results. This spectroscopy led Gell-Mann and Zweig ${ }^{11}$ to postulate that all these particles were made up of more fundamental constituents, namely quarks (or aces). The spectroscopy is then very straightforward--namely all the then known particles were made of three fundamental quarks u,d,s. In particular then the mesons were composed of a quark anti-quark pair and the baryons of three quarks. As such

Meson $3 \times \overline{3}=1+8$

Baryons $3 \times 3 \times 3=1+8+8+10$

Furthermore, all the spin parities of the multiplets can be divided by considering the mesons and baryons as loosely bound states of quarks. A new ingredient was needed and that was the concept of color, that is quarks came in three colors and the observed states were color neutral. Why this worked required the development of QCD and the advent of assymptotic freedom and infrared slavery. As you will shortly hear, the experimental activities involving deeply 
inelastic electron scattering demonstrated that the proton was not fundamental but had hard constituents, partons. This beautiful program complemented later by neutrino scattering elucidated the dynamics of these processes again leading to QCD. Quarks are partons and partons are quarks and all these studies have given us the Standard Model with all its beauty and limitations.

I conclude my talk with a brief discussion of baryon charm. Charm was introduced from both symmetry considerations, four leptons with two families should be accompanied by four quarks of two families as well to fix the rates of certain $\mathrm{K}$ decays. This imposed a particular scheme ${ }^{12}$ (GIM mechanism) which had the quark couplings (dc $\sin \theta)$ and $(\operatorname{cs} \cos \theta)$ where $\theta$ is the Cabibbo angle. As such it was noted that in neutrino interactions on a nucleon, one could produce a single strange particle (in contrast to the normal expected associated production of strange particles) but at a rate corresponding to $\left(\sin ^{2} \theta\right)$. This is illustrated in Fig. 9. As such there are several signatures; opposite sign dileptons $\left(\mu^{-} l^{+}\right)$; opposite sign dileptons accompanied by a single strange particle and the most definitive a $\mu^{-}$with only hadrons and a single strange particle. Such an event ${ }^{13}$ was indeed found in an experiment involving neutrinos interating on protons in the BNL 7-ft bubble chamber Fig. 10. The event was unique in many ways; kinematically there is nothing missing; the three charged positive tracks are identified as pions, by their interaction, decay and a $\delta$ ray; the $V$ particle is unambiguously $a \Lambda^{\circ}$ and the event was initiated by a $13 \mathrm{GeV} / \mathrm{c}$ neutrino. Since the event occurs in hydrogen with a mandatory $\mu^{\circ}$ the hadronic component is doubly postively charged.

The interpretation of the event was

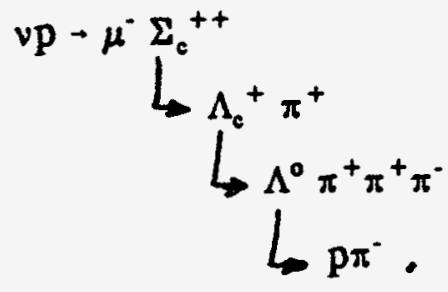

The $\left(\Sigma_{c}^{++}-\Lambda_{c}^{+}\right)$mass difference was measured to be $166 \pm 15 \mathrm{MeV}$ in 
remarkable accord with the estimate by DeRujula, Georgi and Glashow. ${ }^{14}$ The $\Lambda_{c}{ }^{+}$mass was determined to be $2260 \pm 20 \mathrm{MeV}$. All these values arrived at with a new bubble chamber, new optics, beam, etc. A re-evaluation with the chamber systematics in hand yielded $2272 \pm 12 \mathrm{MeV}$, both these values in good agreement with the latest compilation for the mass of $2285 \mathrm{MeV}$. In fact this one event contained evidence for the discovery of two new particles, the $\Sigma_{c}{ }^{++}$and the $\Lambda_{c}{ }^{+}$! Parenthetically, I should remark that I introduced the notation for these baryon charm events in this publication as well as more extensively at the Toronto Conference in $1976 .{ }^{15}$ The question is why wasn't this evidence for charm immediately believed. We believed it as well as Glashow and collaborators and others but what about the rest of the community? There are several observations that I can make on this question. First, unlike the $\Omega^{-}$which was also one event, the concept of charm and any such spectroscopy was not accepted nor in evidence in contrast to many strange particles and the accompanying systematics in the strange arena. Second, the CERN BEBC experiment was not as fortunate as we were and did not observe any such events. Third, and most important, the evidence from SLAC-SPEAR showed no excess of strange particle production at the higher energies. As you heard at this conference, this was later rectified by the efforts of $\mathrm{G}$. Goldhaber who pushed the particle identification limits and observed the charm mesons, $D, D^{*}$ in all their glory. Looking back it was a beautiful event and it was right. In conclusion, I would note that it is important in one's career to be lucky--both in where one happens to be and the times. For me the 1950's and 1960's were great times and BNL and Columbia great places. It is equally important to work with smart people--preferentially smarter than oneself and I had such a pleasure in my efforts with Jack Steinberger, Mel Schwartz, and Bob Palmer. And finally, it is crucial to talk and to listen to smart people and to pick them correctly. Again, I have benefited enormously from such interactions with T.D. Lee, Murray Gell-Mann and Shelly Glashow. 


\section{REFERENCES}

1. Anderson et al., PR 91, 155, 1953.

2. Erwin et al., PRL 6, 628, 1961.

3. Alston et al., PRL 5, 520, 1960.

4. Alston et al., PRL 6, 300, 1961.

5. Maglic et al., PRL 7, 178, 1961.

6. Pevsner et al., PRL 7, 421, 1961.

7. Bertanza et al., PRL 9, 180, 1962.

8. Goldberg et al., PRL 12, 549, 1964.

9. Gell-Mann, Cal. Inst. Tech. Report CTSR-20, 1961.

10. Barnes et al., PRL 12, 204, 1964.

11. Gell-Mann, Phys. Letters 8, 214, 1964.

Zweig, CERN Report No. 8181/Th 401 and No. 8419/Th 412, 1964.

12. Glashow, lliopoulos and Marani, (G.I.M.) PRD 2, 1285, 1970.

13. Cazzoli et al., PRL 1125, 1975.

14. De Rujula, et al., PRD 2, 1285, 1970.

15. Samios, Proc. of the IVth Int. conf. on Baryon Resonances (Toronto, 1980), p. 309. 


\section{FIGURE CAPTIONS}

Fig. 1 Formation and production experiments.

Fig. 2 Total $\pi^{+} p$ and $\pi^{-}$p cross sections as a function of energy.

Fig. 3 Evidence for the $\Sigma(1385)$.

Fig. 4 Evidence for the $\eta(550)$.

Fig. 5 Evidence for the $\varphi(1020)$.

Fig. 6 Evidence for the $\Xi(1535)$.

Fig. 7 Evidence for the $\Omega(1675)$.

Fig. 8 Continuing evidence for the $\Omega^{-}(1675)$.

Fig. 9 Charm and the G.I.M. mechanism.

Fig. 10 Evidence for baryon charm, $\Sigma_{c}^{++}$and $\Lambda_{c}{ }^{+}$.

Table I Possible resonances of strongly interacting particles (as of August 1961).

\section{DISCLAIMER}

This report was prepared as an account of work sponsored by an agency of the United States Government. Neither the United States Government nor any agency thereof, nor any of their employees, makes any warranty, express or implied, or assumes any legal liability or responsibility for the accuracy, completeness, or usefulness of any information, apparatus, product, or process disclosed, or represents that its use would not infringe privately owned rights. Reference herein to any specific commercial product, process, or service by trade name, trademark, manufacturer, or otherwise does not necessarily constitute or imply its endorsement, recommendation, or favoring by the United States Government or any agency thereof. The views and opinions of authors expressed herein do not necessarily state or reflect those of the United States Government or any agency thereof. 
Formation Experiments

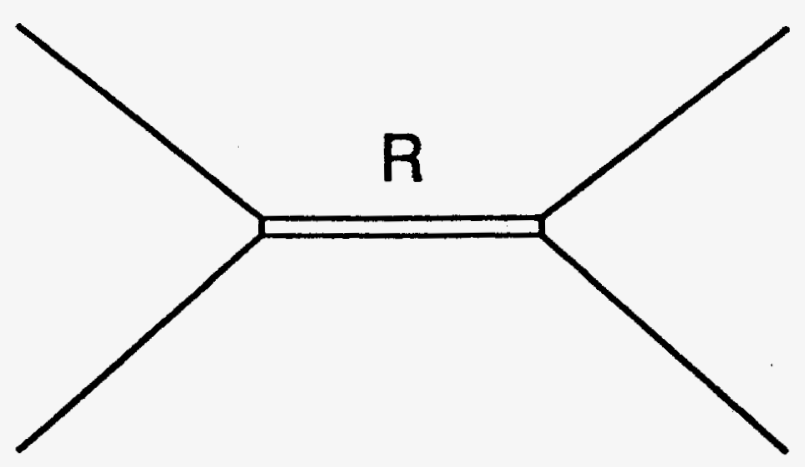

\section{Production Experiments}

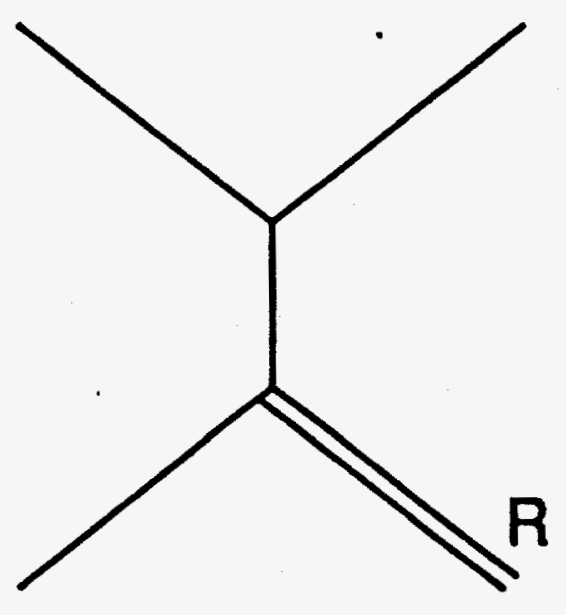

Fig. 1 
PLOTS OF CROSS SECTIONS AND RELATED QUANTITIES

Hadronic Cross Sections
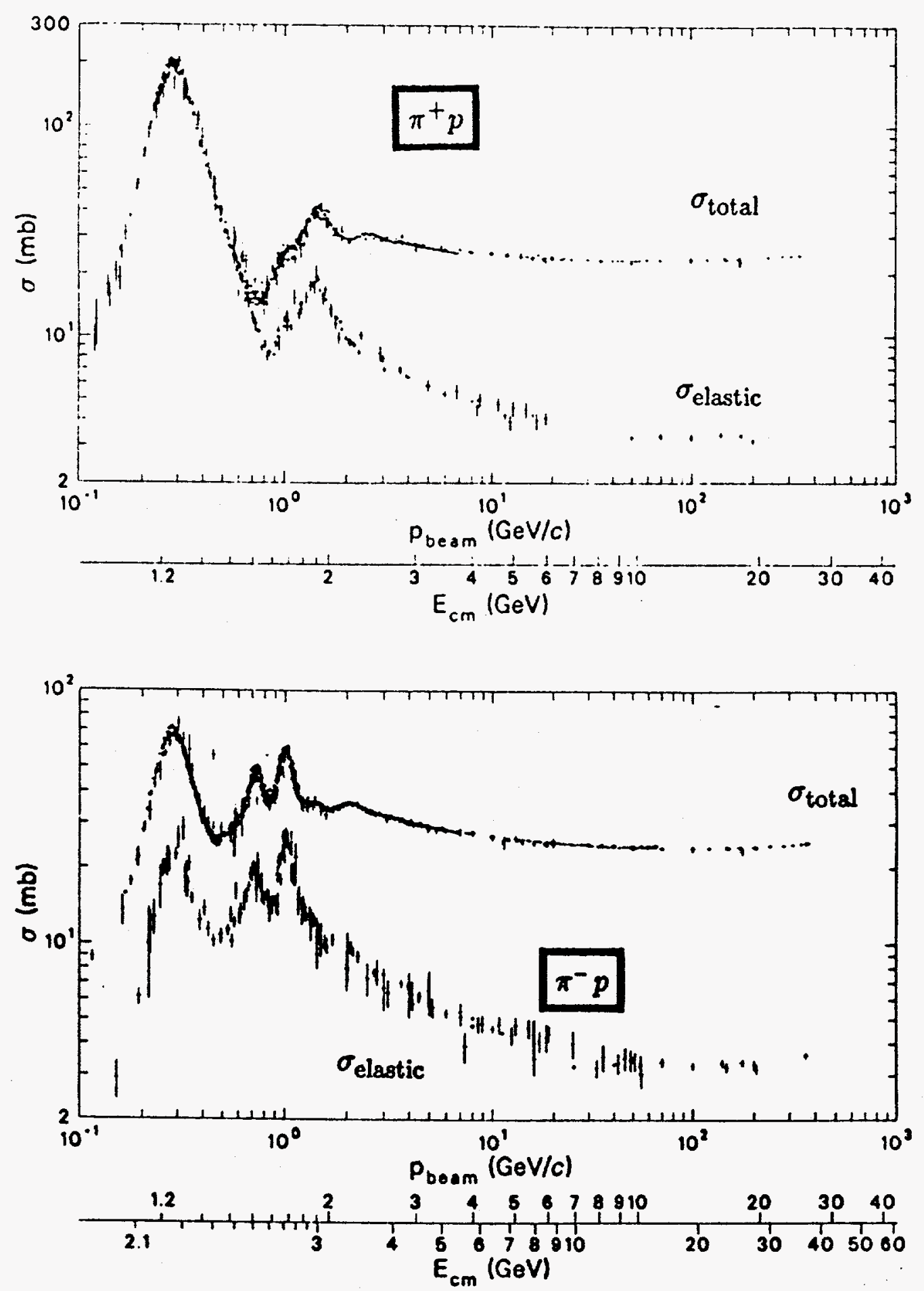

Fig. 2 


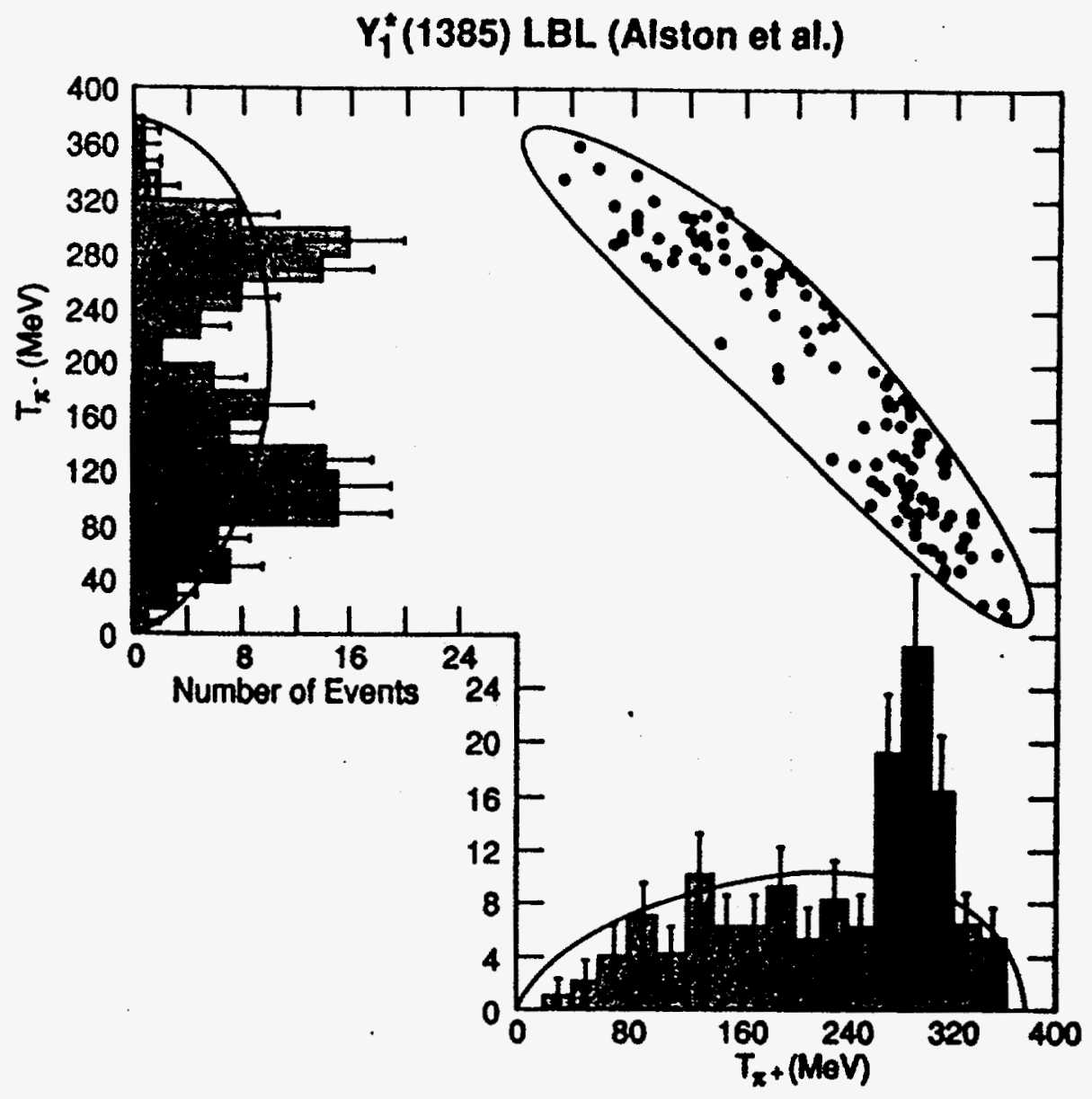

Fig. 3 


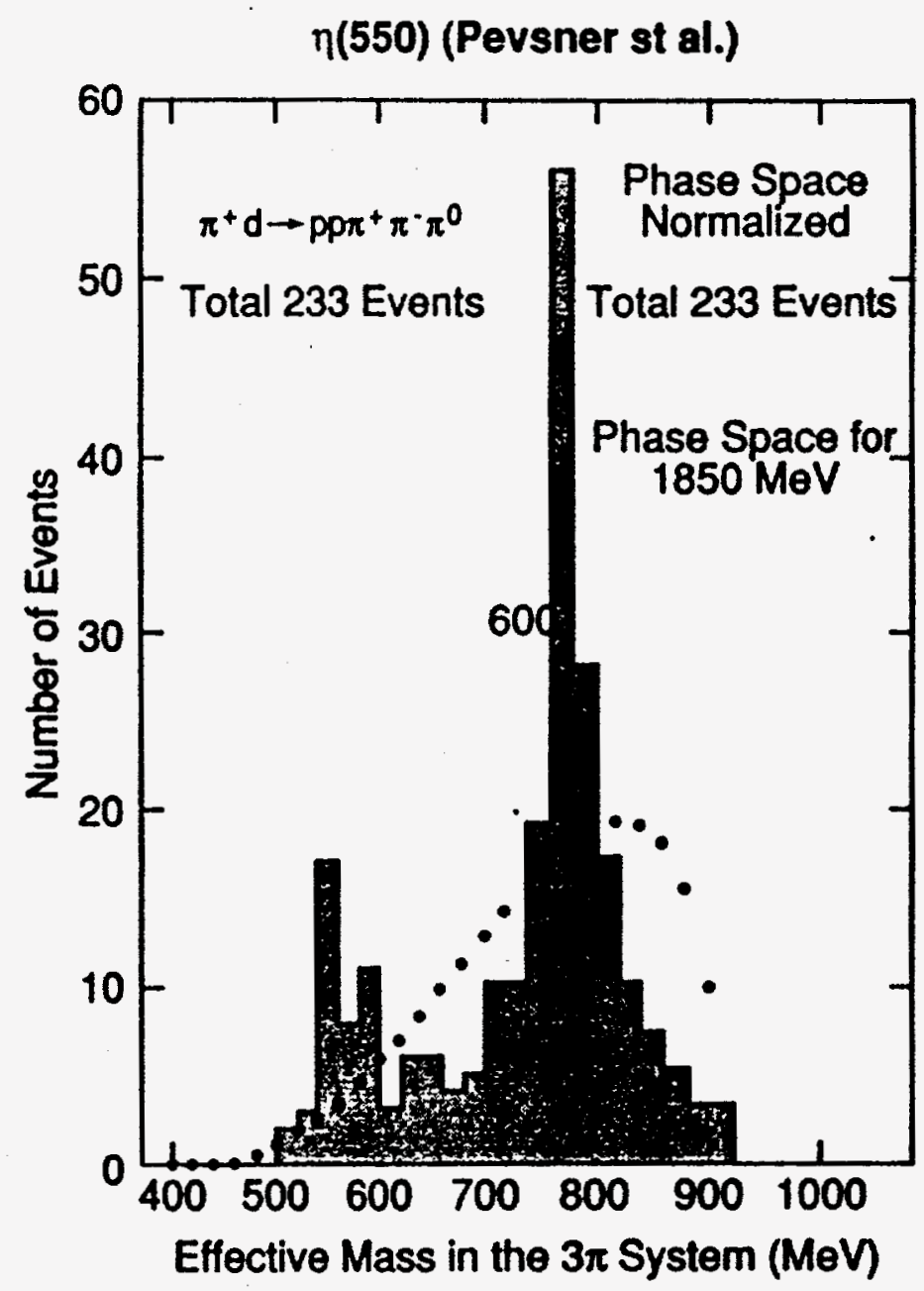

Fig. 4 


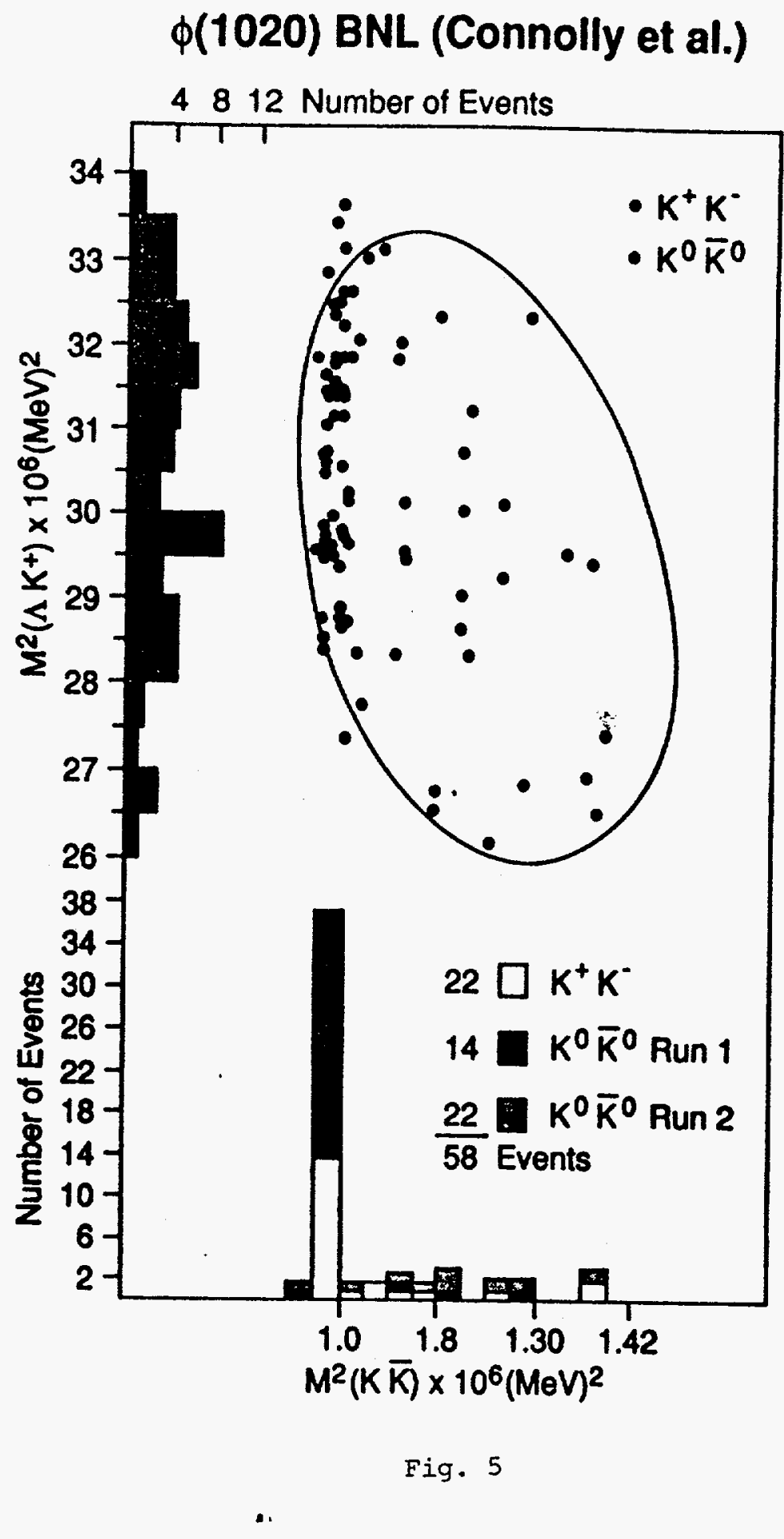


$\Xi$ "(1530) BNL (Bertanza et al.)

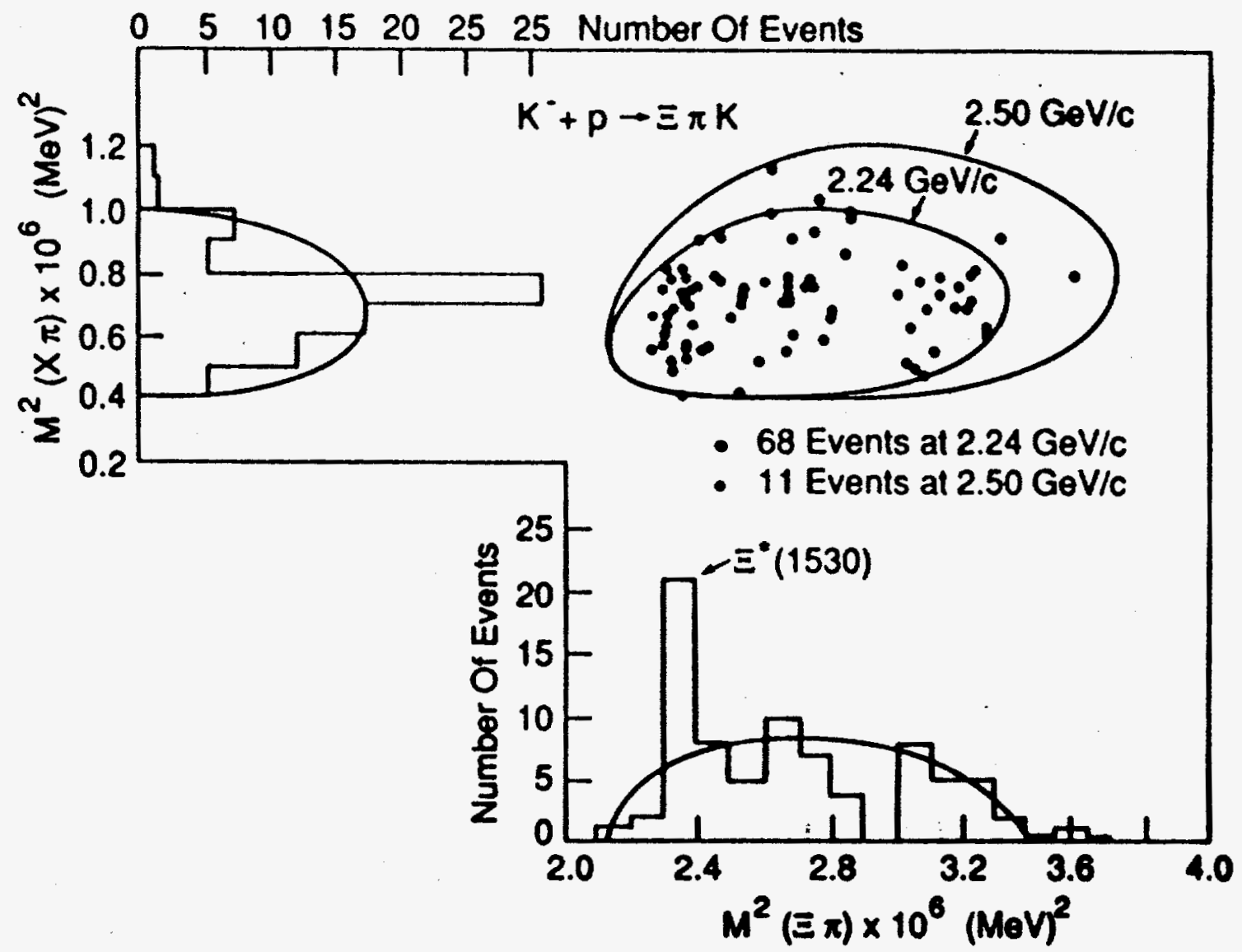

Fig. 6 The Dalitz plot for the channel $\Xi \pi K$ projected on the $M^{2}(K \pi)$ and the $M^{2}(\Xi \pi)$ axes. The solid curves on the projections are the invariant phasespace curves normalized to the total number of events. 


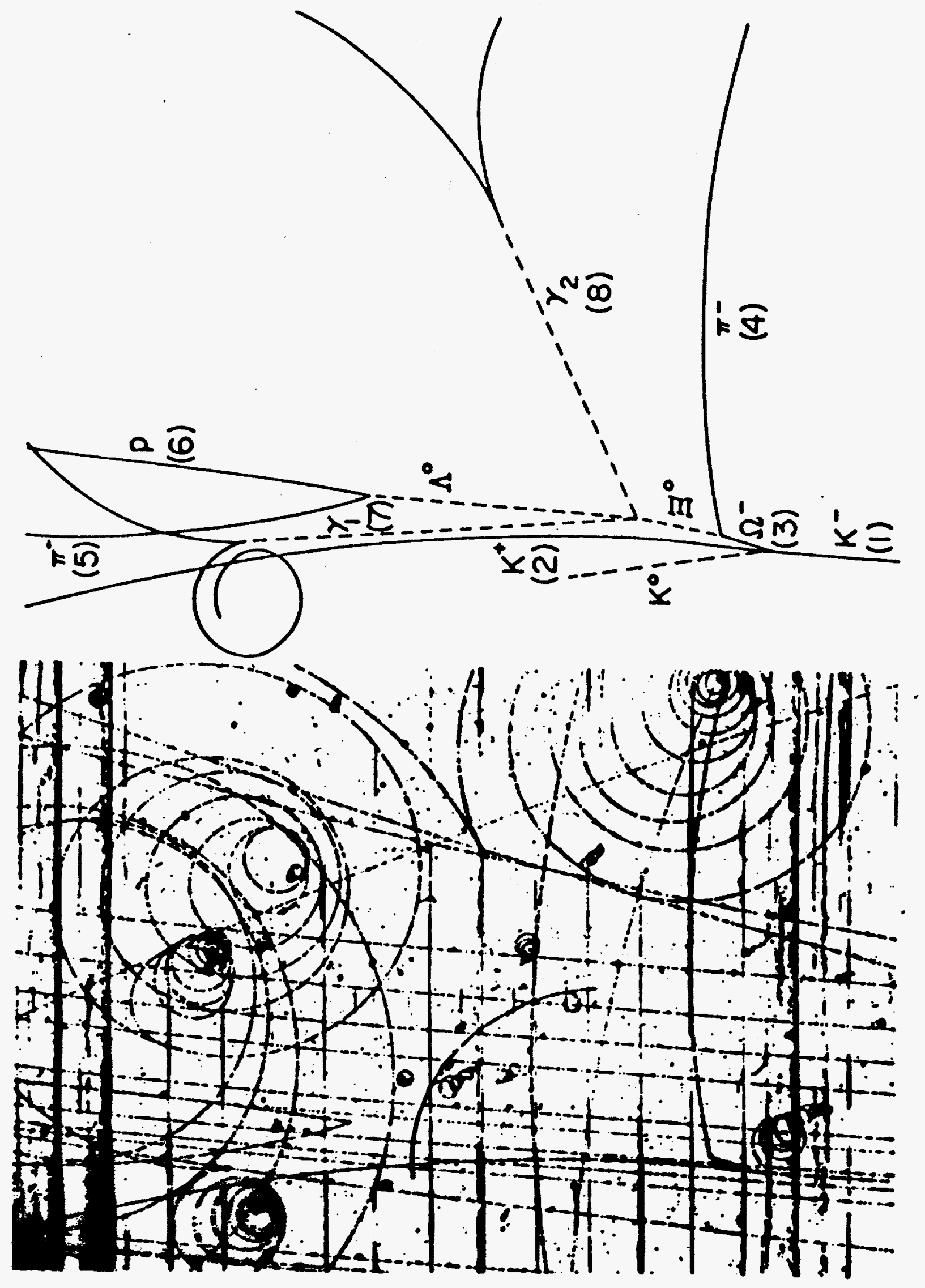

i 


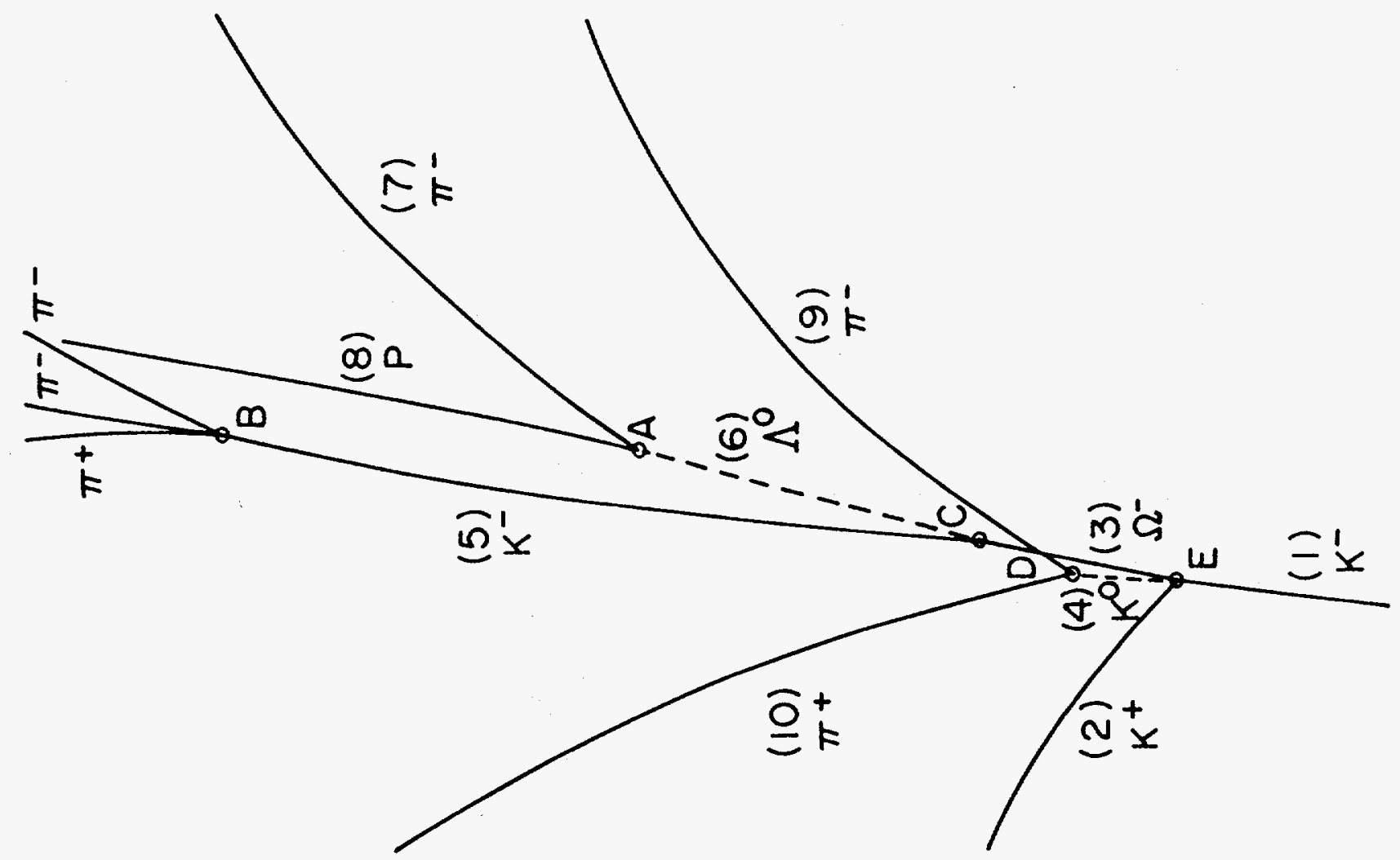

$\infty$
$\dot{\theta}$
$\dot{\theta}=1$

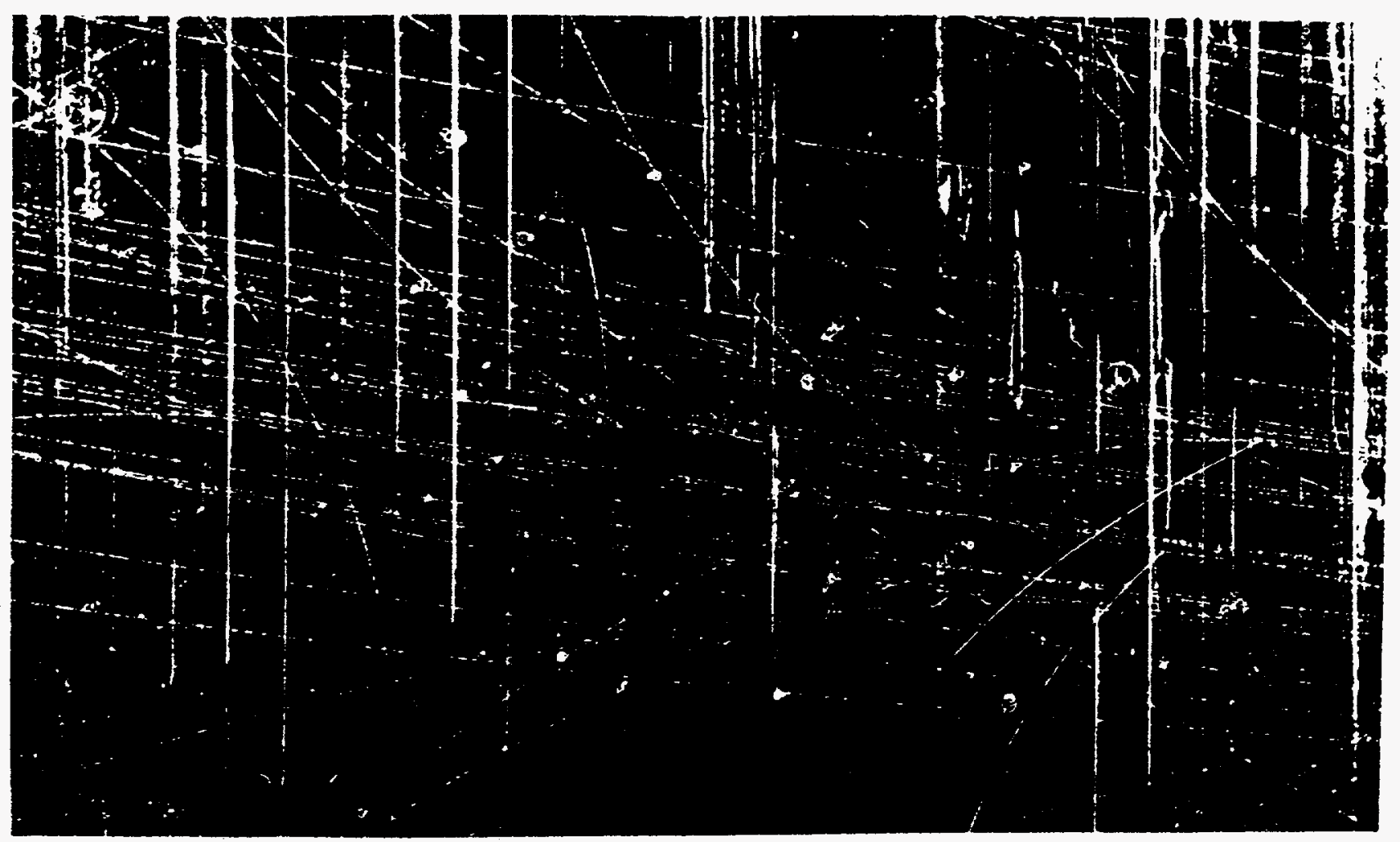



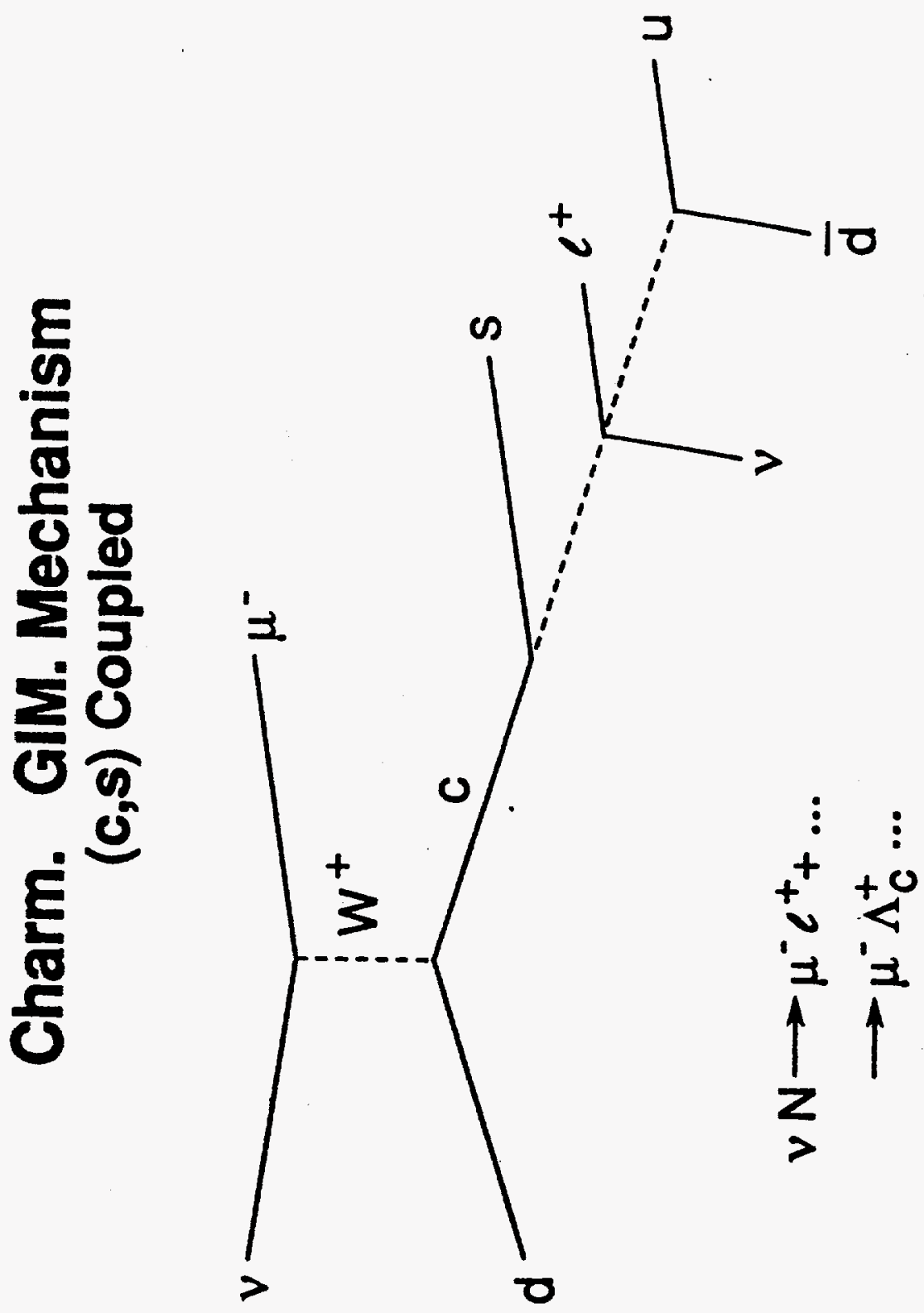

$\underset{\substack{a \\ \dot{a}}}{\sigma}$ 

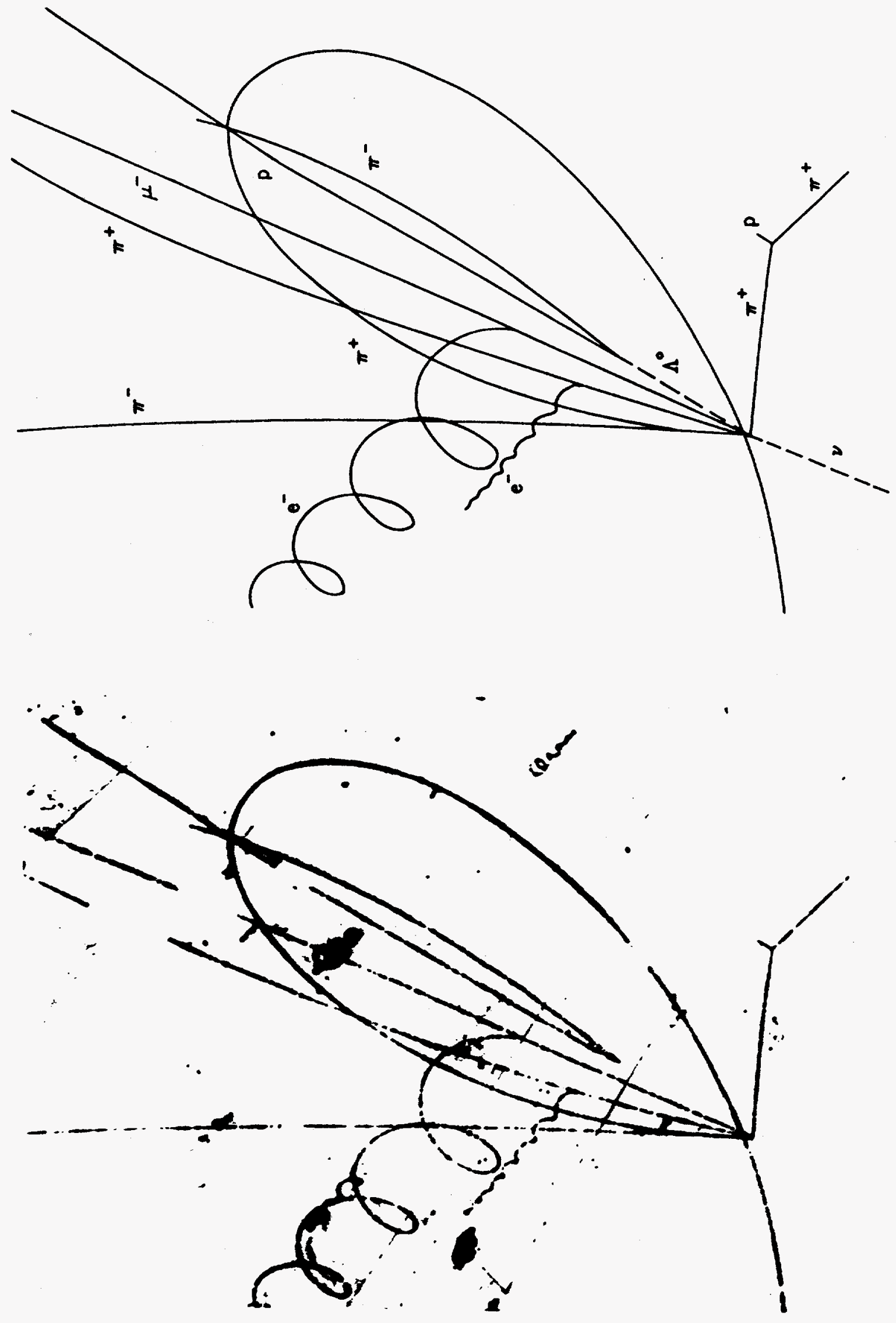
Possible resonances of strongly interacting particles (as of August 1961)

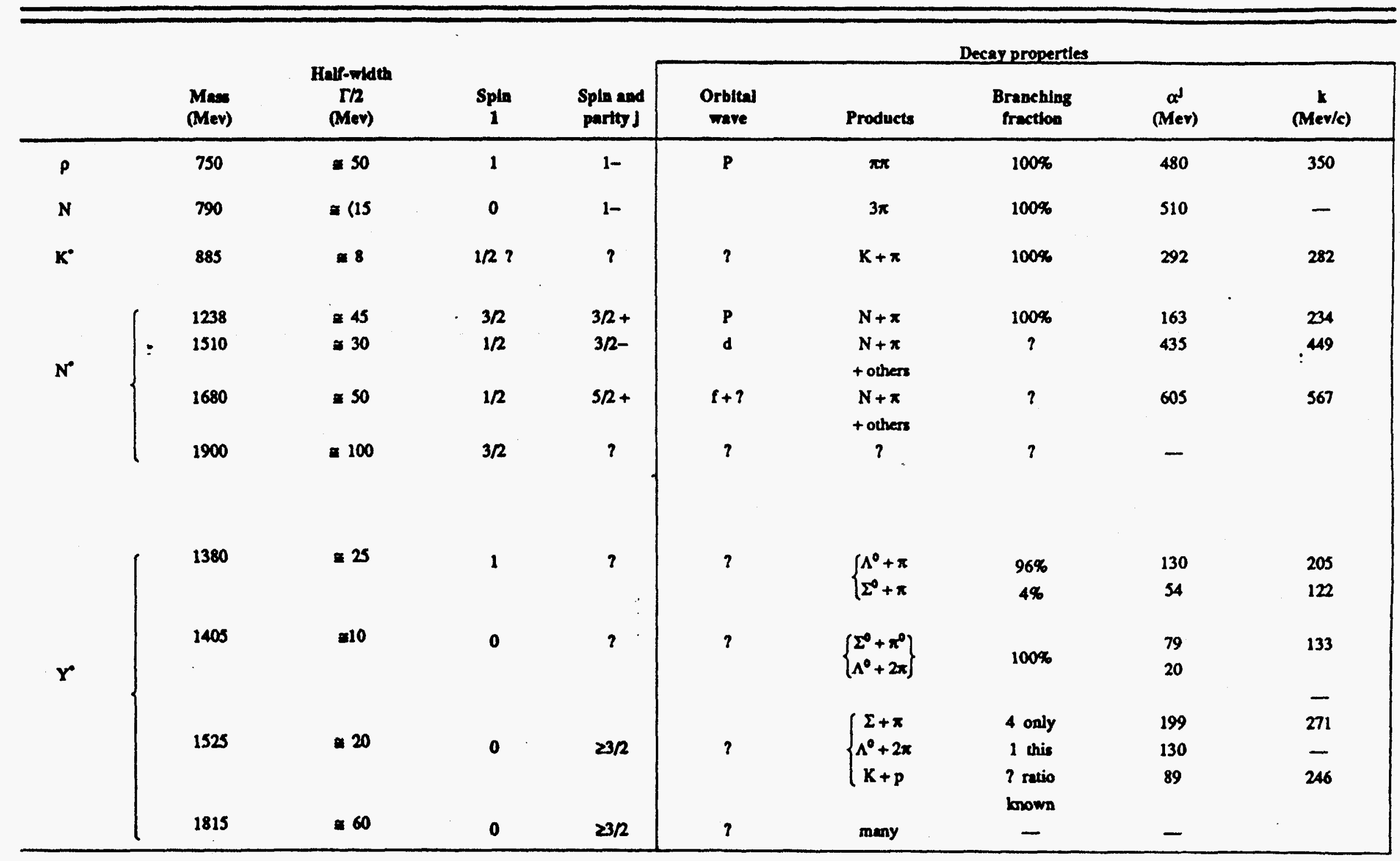

TABLE I 